\title{
Estimating heavy-tail exponents through max self-similarity
}

Stilian Stoev (sstoev@umich.edu)

Department of Statistics

University of Michigan

\author{
Probability \& Statistics Colloquium \\ Michigan State University, October, 2006
}

Joint work with George Michailidis and Murad S. Taqqu 


\section{Part I: Motivation}




\section{Heavy tailed data}

- A random variable $X$ is said to be heavy-tailed if

$$
\mathbb{P}\{|X| \geq x\} \sim L(x) x^{-\alpha}, \quad \text { as } x \rightarrow \infty,
$$

for some $\alpha>0$ and a slowly varying function $L$.

o Here we focus on the simpler but important context:

$X \geq 0$, a.s. and $\mathbb{P}\{X>x\} \sim C x^{-\alpha}$, as $x \rightarrow \infty$.

$\circ X$ (infinite moments) For $p>0$,

$$
\mathbb{E} X^{p}<\infty \quad \text { if and only if } \quad p<\alpha .
$$

In particular,

$$
0<\alpha \leq 2 \Rightarrow \operatorname{Var}(X)=\infty
$$

and

$$
0<\alpha \leq 1 \quad \Rightarrow \quad \mathbb{E}|X|=\infty
$$

- The estimation of the heavy-tail exponent $\alpha$ is an important problem with rich history.

- Why do we need heavy-tail models?

Every finite sample $X_{1}, \ldots, X_{n}$ has finite sample mean, variance and all sample moments!

Why consider heavy tailed models in practice?! 


\section{Why use heavy-tailed models?}

"All models are wrong, but some are useful."

George Box

Let $F$ and $G$ be any two distributions with positive densities on $(0, \infty)$.

Let $\epsilon>0$ and $x_{1}, \ldots, x_{n} \in(0, \infty)$ be arbitrary, then both:

$$
\mathbb{P}_{F}\left\{X_{i} \in\left(x_{i}-\epsilon, x_{i}+\epsilon\right), i=1, \ldots, n\right\}>0
$$

and

$$
\mathbb{P}_{G}\left\{X_{i} \in\left(x_{i}-\epsilon, x_{i}+\epsilon\right), i=1, \ldots, n\right\}>0
$$

are positive!

- For a given sample, very many models apply.

- The ones that continue to work as the sample grows are most suitable.

We next present real data sets of Financial, Insurance and Internet data. They can be very heavy tailed. 


\section{Traded volumes on the Intel stock}
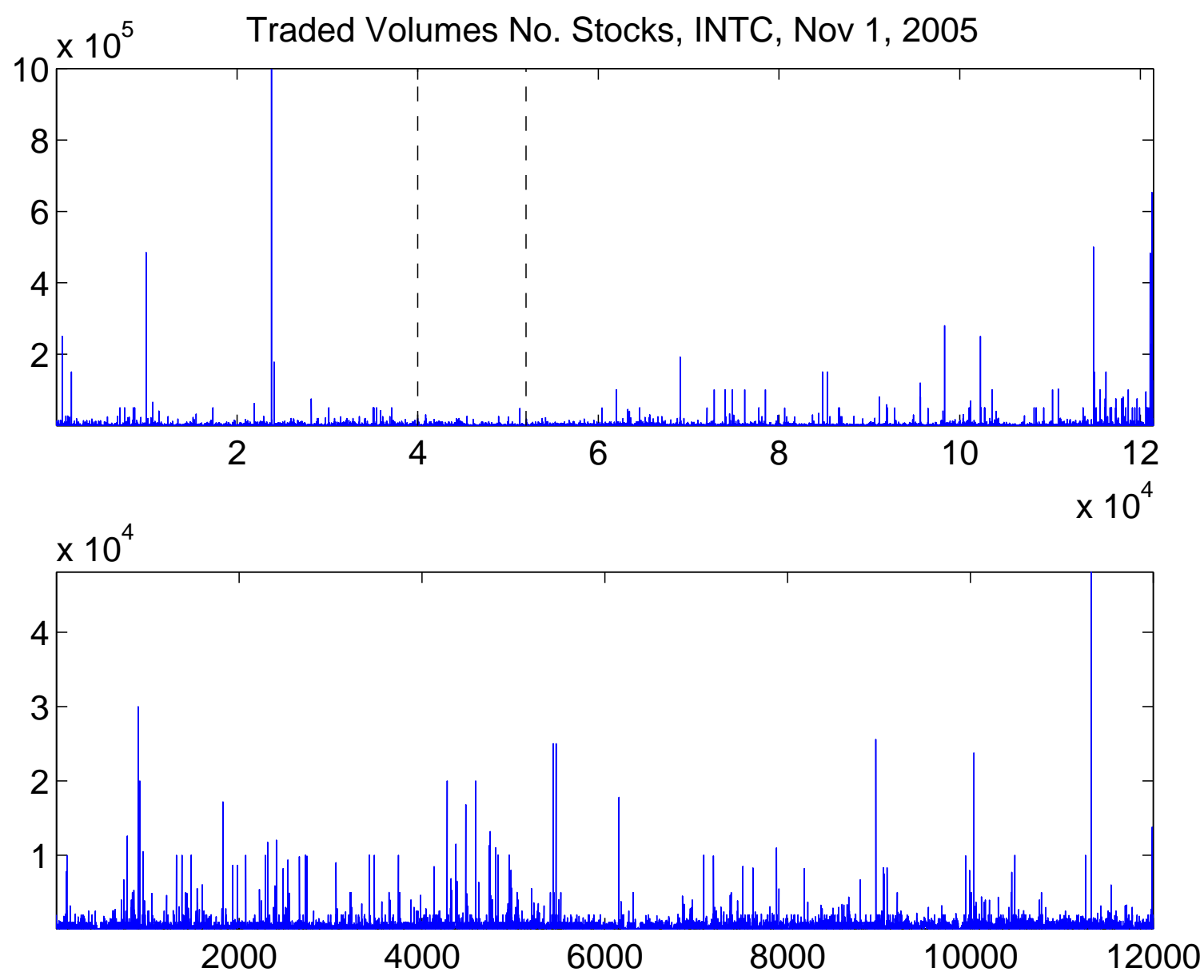


\section{Insurance claims due to fire loss}
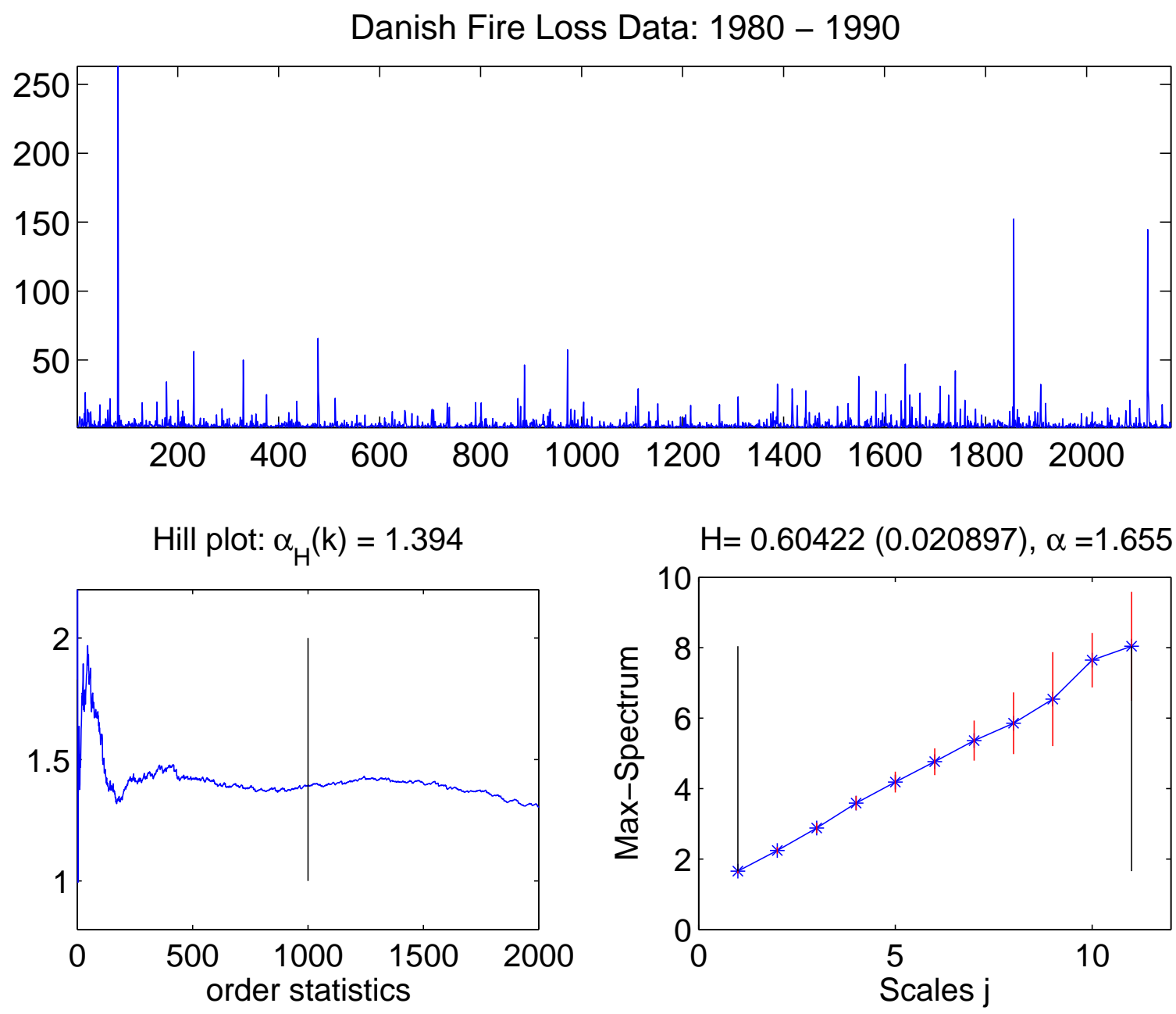


\section{TCP flow sizes (in number of packets)}

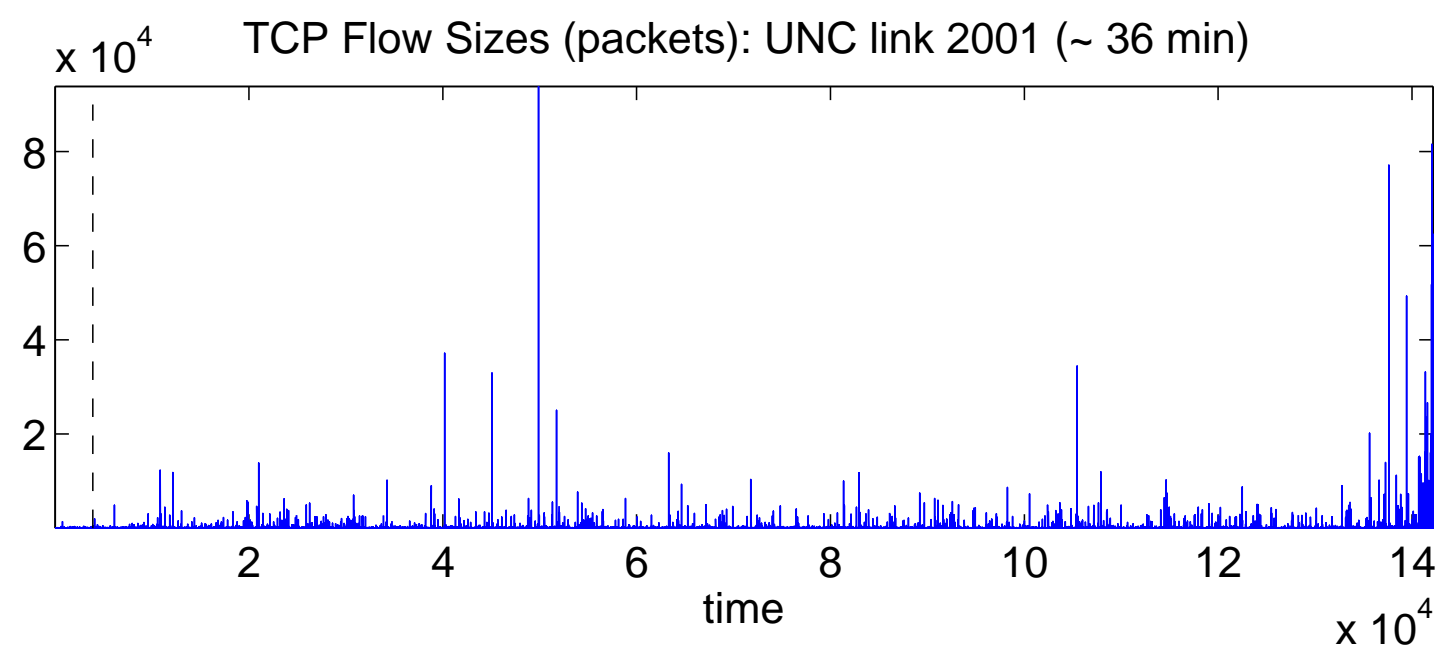

The first minute

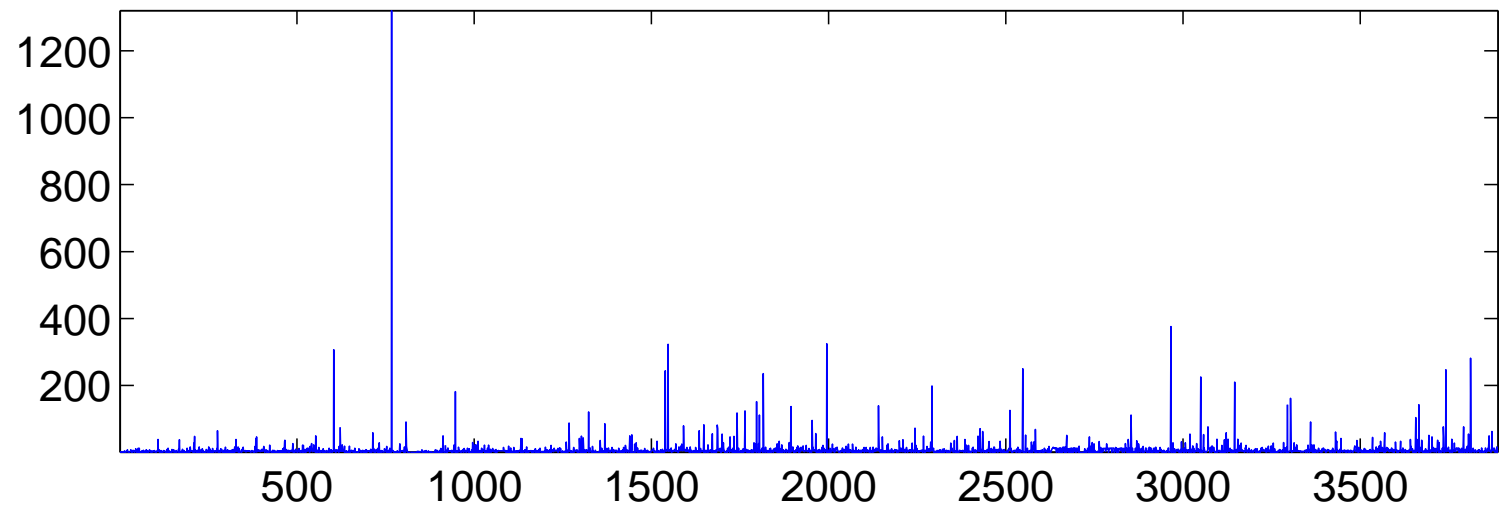




\section{History}

- Hill (1975) worked out the MLE in the Pareto model $\mathbb{P}\{X>x\}=x^{-\alpha}, x \geq 1$ and introduced the Hill plot:

$$
\widehat{\alpha}_{H}(k):=\left(\frac{1}{k} \sum_{i=1}^{k} \log \left(X_{i, n}\right)-\log \left(X_{k+1, n}\right)\right)^{-1},
$$

where $X_{1, n} \geq X_{2, n} \geq \cdots \geq X_{k, n}$ are the top-K order statistics of the sample.

- How to choose $k$ ?

o pick $k$ where the plot of $\widehat{\alpha}_{H}(k)$ vs. $k$ stabilizes.

o serious problems in practice:

volatile \& hard to interpret: "Hill horror plot"

confidence intervals

robustness

- Consistency and asymptotic normality resolved: Weissman (1978), Hall (1982) in semi-parametric setting.

- Many other estimators: kernel based Csörgő, Deheuvels and Mason (1985), moment Dekkers, Einmahl and de Haan (1989), among many others.

- Most estimators exploit the top order statistics. 


\section{Hill horror plots: TCP flow sizes}
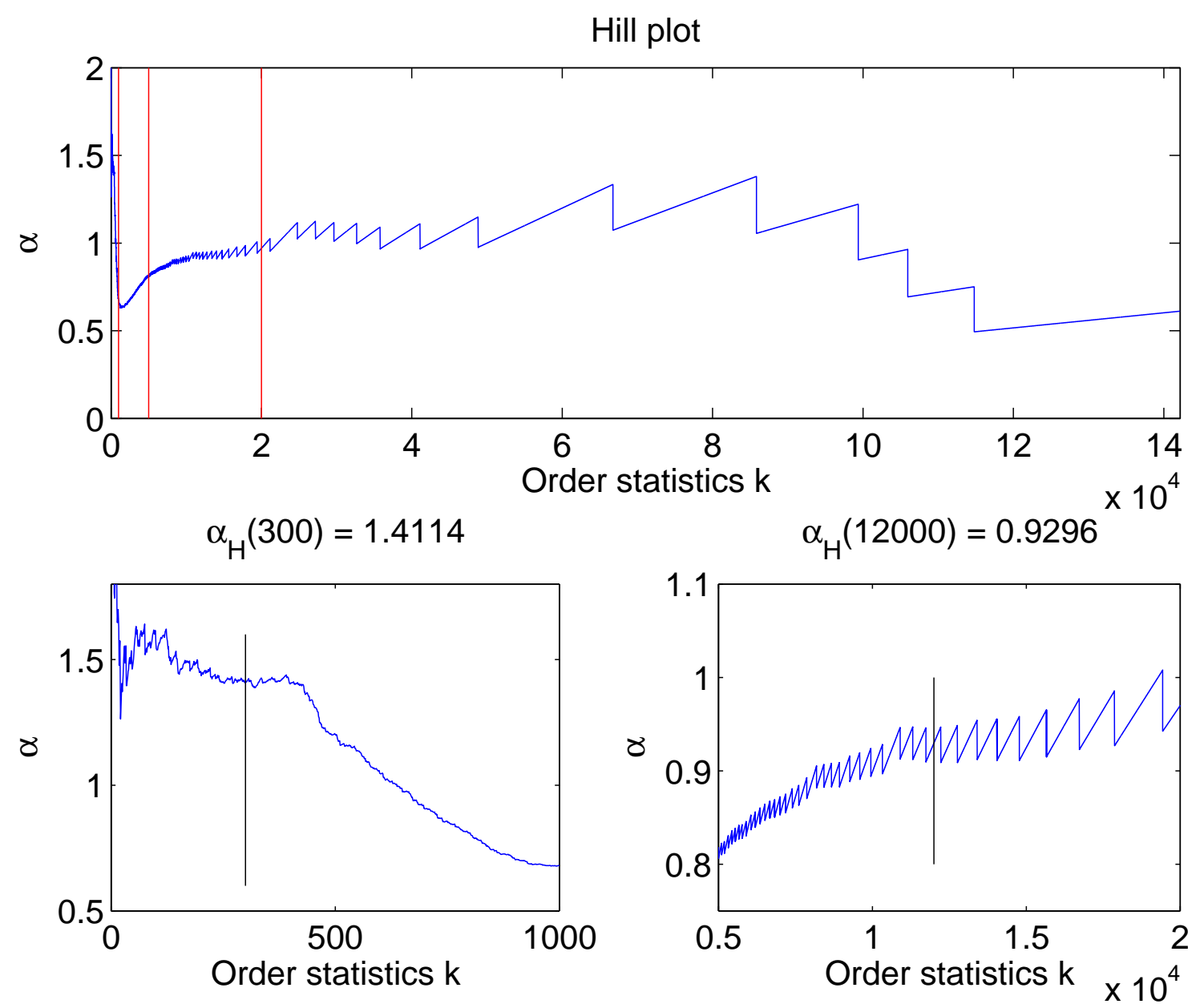

9 


\section{Part II: Max self-similarity \& max-spectrum}




\section{Fréchet max-stable laws}

Consider i.i.d. $X_{i}$ 's with $\mathbb{P}\left\{X_{1}>x\right\} \sim C x^{-\alpha}, x \rightarrow \infty$. By the Fisher-Tippett-Gnedenko theorem:

$$
\frac{1}{n^{1 / \alpha}} \max _{1 \leq i \leq n} X_{i} \equiv \frac{1}{n^{1 / \alpha}} \bigvee_{i=1}^{n} X_{i} \stackrel{d}{\longrightarrow} Z, \quad \text { as } n \rightarrow \infty,
$$

where $Z$ is $\alpha$-Fréchet extreme value distribution:

$$
\mathbb{P}\{Z \leq x\}=\exp \left\{-C x^{-\alpha}\right\}, \quad x>0 .
$$

- The extreme value distributions are max-stable. In particular, for i.i.d. $\alpha$-Fréchet $Z, \& Z_{i}$ 's:

$$
Z_{1} \vee \cdots \vee Z_{n} \stackrel{d}{=} n^{1 / \alpha} Z
$$

- A time series of i.i.d. $\alpha$-Fréchet $\left\{Z_{k}\right\}$ is $1 / \alpha$-max-selfsimilar:

$$
\forall m \in \mathbb{N}:\left\{\vee_{1 \leq i \leq m} Z_{m(k-1)+i}\right\}_{k \in \mathbb{N}} \stackrel{d}{=} m^{1 / \alpha}\left\{Z_{k}\right\}_{k \in \mathbb{N}} .
$$

- Block-maxima of size $m$ have the same distribution as the original data, rescaled by the factor $m^{1 / \alpha}$.

- Any heavy-tailed $\left\{X_{k}\right\}$ (i.i.d.) data set is asymptotically max self-similar. 


\section{Max-spectrum}

Given a positive sample $X_{1}, \ldots, X_{n}$, consider the dyadic block-maxima:

$$
D(j, k):=\max _{1 \leq i \leq 2^{j}} X_{2^{j}(k-1)+i} \equiv \bigvee_{i=1}^{2^{j}} X_{2^{j}(k-1)+i}
$$

with $k=1, \ldots, n_{j}=\left[n / 2^{j}\right]$.

- In view of the asymptotic scaling:

$$
\frac{1}{2^{j / \alpha}} D(j, k) \stackrel{d}{\longrightarrow} Z, \quad(j \rightarrow \infty),
$$

observe that

$$
Y_{j}:=\frac{1}{n_{j}} \sum_{j=1}^{n_{j}} \log _{2} D(j, k) \simeq j / \alpha+c, \quad\left(j, n_{j} \rightarrow \infty\right)
$$

where $c:=\mathbb{E} \log _{2} Z$.

- The last asymptotics "follow" from the LLN since $D(j, k)$ 's are independent in $k$.

- The max-spectrum of the data is defined as the statistics:

$$
Y_{j}, j=1, \ldots,\left[\log _{2}(n)\right] .
$$

- Can identify $\alpha$ from the slope of the $Y_{j}$ 's vs. $j$, for large $j$ 's. 


\section{Max-spectrum estimators of $\alpha$}

Given the max-spectrum $Y_{j}, j=1, \ldots,\left[\log _{2}(n)\right]$, define

$$
\widehat{\alpha}\left(j_{1}, j_{2}\right):=\left(\sum_{j=j_{1}}^{j_{2}} w_{j} Y_{j}\right)^{-1},
$$

where $\sum_{j} j w_{j}=1$ and $\sum_{j} w_{j}=0$.

- That is, use linear regression to estimate the slope $1 / \alpha$.

- Issues:

oweighted or generalized least squares must be used, since

$$
\operatorname{Var}\left(Y_{j}\right) \propto 1 / n_{j} \propto 2^{j}
$$

- The choice of the scales $j_{1}$ and $j_{2}$ is critical.

- These issues and confidence intervals, are addressed in Stoev, Michailidis and Taqqu (2006). 


\section{Examples of max-spectra}
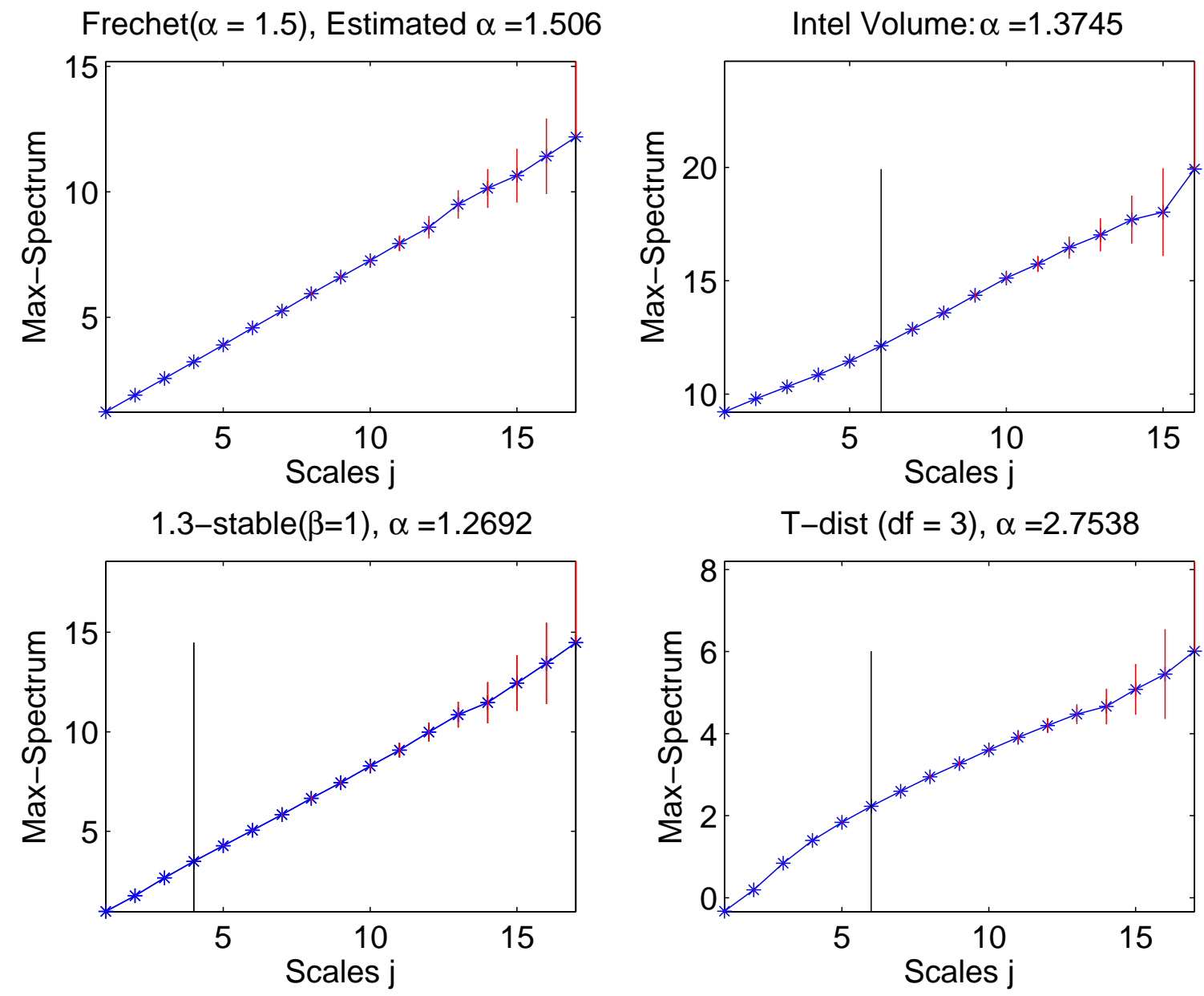


\section{Robustness of the max-spectrum}

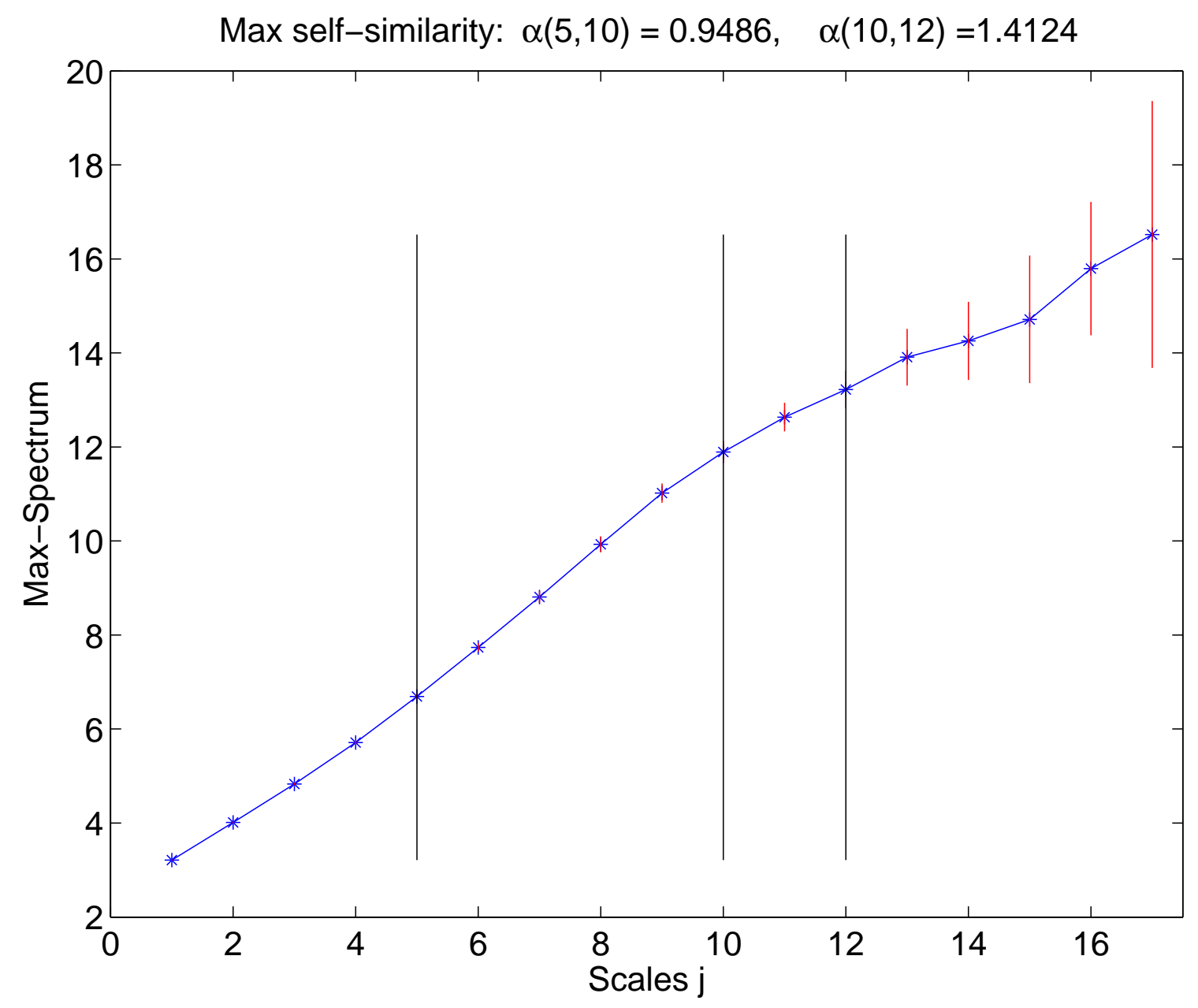

Compare with the Hill horror plot of the Internet flowsize data (next slide). 
Hill horror plots: TCP flow sizes
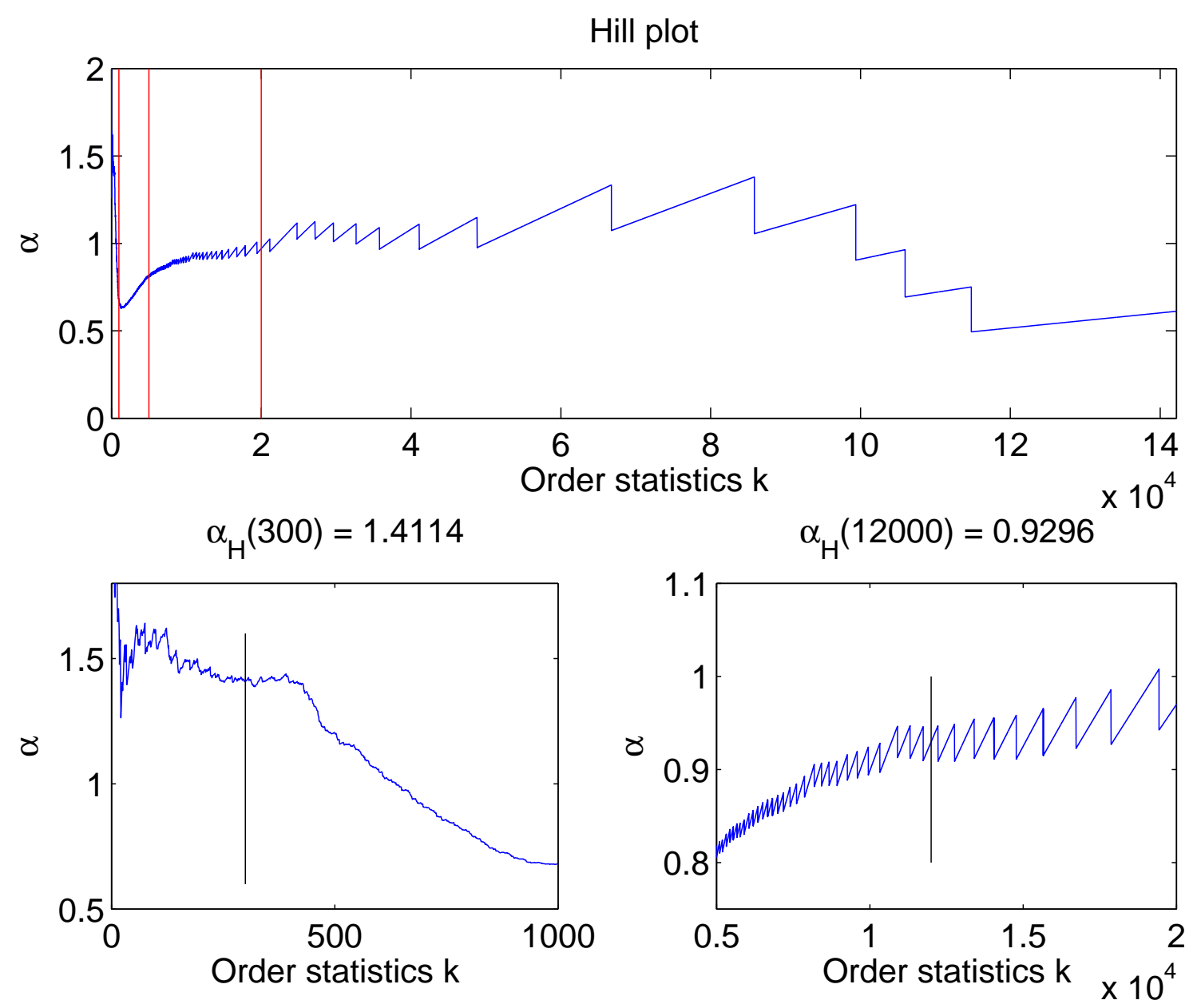

Compare with the max-spectrum plot of the Internet flow-size data (previous slide). 
Part III: Asymptotic results 


\section{Asymptotic normality of the max-spectrum}

Let $\mathbb{P}\left\{X_{k} \leq x\right\}=: F(x)$, where

$$
1-F(x)=C x^{-\alpha}\left(1+\mathcal{O}\left(x^{-\beta}\right)\right), \quad(x \rightarrow \infty),
$$

with some $\beta>0$.

- Consider the max-spectrum $\left\{Y_{j}\right\}$ for the range of scales:

$$
r(n)+j_{1} \leq j \leq r(n)+j_{2},
$$

with fixed $j_{1}<j_{2}$ and $r(n) \rightarrow \infty, n \rightarrow \infty$.

Theorem Under (1) and another technical condition,

$$
\begin{aligned}
\sup _{x \in \mathbb{R}} \mid \mathbb{P} & \left\{\sqrt{n_{j_{2}+r}}\left(\left(\vec{\theta}, \vec{Y}_{r}\right)-\left(\vec{\theta}, \vec{\mu}_{r}\right)\right) \leq x\right\}-\Phi\left(x / \sigma_{\vec{\theta}}\right) \mid \\
& \leq C_{\vec{\theta}}\left(1 / 2^{r \beta / \alpha}+r 2^{r / 2} / \sqrt{n}\right),
\end{aligned}
$$

where $\Phi$ is the standard Normal c.d.f.

Here $\vec{Y}_{r}=\left(Y_{r+j}\right)_{j=j_{1}}^{j_{2}}$ and

$$
\left(\vec{\theta}, \vec{Y}_{r}\right)=\sum_{j=j_{1}}^{j_{2}} \theta_{j} Y_{r+j}, \quad \mu_{r}(j)=(r+j) / \alpha+c
$$

and

$$
\sigma_{\vec{\theta}}^{2}=\left(\vec{\theta}, \Sigma_{\alpha} \vec{\theta}\right):=\sum_{i, j=j_{1}}^{j_{2}} \theta_{i} \Sigma_{\alpha}(i, j) \theta_{j}>0
$$




\section{The covariance structure of the max-spectrum}

- The asymptotic covariance matrix $\Sigma$ is the same as if the data were i.i.d. $\alpha$-Fréchet!

- The intuition is that the block-maxima

$$
\left\{D(r+j, k), k=1, \ldots, n_{r+j}\right\}
$$

behave like i.i.d. $\alpha$-Fréchet variables, as $r=r(n) \rightarrow \infty$.

- The covariance entries are given by:

$$
\Sigma_{\alpha}(i, j)=\alpha^{-2} 2^{i \vee j-j_{2}} \psi(|i-j|),
$$

with

$$
\psi(a):=\operatorname{Cov}\left(\log _{2}\left(Z_{1}\right), \log _{2}\left(Z_{1} \vee\left(2^{a}-1\right) Z_{2}\right), \quad a \geq 0,\right.
$$

for i.i.d. $1-$ Fréchet $Z_{1} \& Z_{2}$.

- Note that $\alpha$ appears only as a factor in $\Sigma_{\alpha}$ :

$$
\Sigma_{\alpha}=\alpha^{-2} \Sigma_{1}
$$

It does not affect the correlation structure of the maxspectrum.

- GLS estimators for $\alpha$ use the matrix $\Sigma_{\alpha}$.

- Asymptotic normality for $\widehat{\alpha}\left(r+j_{1}, r+j_{2}\right)$ follows from the last theorem.

- Stoev, Michailidis and Taqqu (2006) has details on confidence intervals for $\alpha$ and automatic selection of $j_{1} \& j_{2}$. 


\section{Rates for moment functionals of maxima}

Let $X_{1}, \ldots, X_{n}$ be i.i.d. from $F$ and recall that

$$
M_{n}:=\frac{1}{n^{1 / \alpha}} \bigvee_{1 \leq i \leq n} X_{i} \stackrel{d}{\longrightarrow} Z, \quad(n \rightarrow \infty) \text {. }
$$

- Are there results on the rate of convergence

$$
\mathbb{E} f\left(M_{n}\right) \longrightarrow \mathbb{E} f(Z), \quad(n \rightarrow \infty)
$$

for "reasonable" f's?

- Pickands (1975) shows only the convergence of moments (no rates).

- Our approach: consider

$$
F(x)=\exp \left\{-\sigma^{\alpha}(x) x^{-\alpha}\right\}, \quad x \in \mathbb{R},
$$

with

$$
\sigma^{\alpha}(x) \longrightarrow C, \quad(x \rightarrow \infty) .
$$

○ Note: $1-F(x) \sim C x^{-\alpha}, x \rightarrow \infty$ is equivalent to (3).

- Extra assumption:

$$
\left|\sigma^{\alpha}(x)-C\right| \leq D x^{-\beta}, \quad \text { for large } x \text {. }
$$




\section{Rates (cont'd)}

But (3) is very convenient to handle rates!

- Note that

$$
\begin{aligned}
\mathbb{P}\left\{M_{n} \leq x\right\} & =\mathbb{P}\left\{X_{1} \leq n^{1 / \alpha} x\right\}^{n}=F\left(n^{1 / \alpha} x\right)^{n} \\
& =\exp \left\{-\sigma^{\alpha}\left(n^{1 / \alpha} x\right) x^{-\alpha}\right\} .
\end{aligned}
$$

- Thus,

$\mathbb{E} f\left(M_{n}\right)=\int_{0}^{\infty} f(x) d F_{n}(x)=\int_{0}^{\infty} f(x) d \exp \left\{-\sigma^{\alpha}\left(n^{1 / \alpha} x\right) x^{-\alpha}\right\}$, and also

$$
\mathbb{E} f(Z)=\int_{0}^{\infty} f(x) d G(x)=\int_{0}^{\infty} f(x) d \exp \left\{-C x^{-\alpha}\right\} .
$$

- Now, integration by parts yields:

$$
\mathbb{E}\left(f\left(M_{n}\right)-f(Z)\right)=\int_{0}^{\infty}\left(G(x)-F_{n}(x)\right) f^{\prime}(x) d x .
$$

o However $G(x)$ and $F_{n}(x)$ are of the same "exponential" form! 


\section{Rates (cont'd)}

By the mean value theorem:

$$
\begin{aligned}
\left|F_{n}(x)-G(x)\right| & =\left|\exp \left\{-\sigma^{\alpha}\left(n^{1 / \alpha} x\right) x^{-\alpha}\right\}-\exp \left\{-C x^{-\alpha}\right\}\right| \\
& \leq\left|\sigma^{\alpha}\left(n^{1 / \alpha} x\right)-C\right| x^{-\alpha} \exp \left\{-c x^{-\alpha}\right\} \\
& \leq D n^{-\beta / \alpha} x^{-(\alpha+\beta)} \exp \left\{-c x^{-\alpha}\right\}, \quad \text { as } x \rightarrow \infty
\end{aligned}
$$

- Thus, for any $\epsilon>0$, as $n \rightarrow \infty$,

$$
\begin{aligned}
& \int_{\epsilon}^{\infty}\left|F_{n}(x)-G(x)\right|\left|f^{\prime}(x)\right| d x \\
& \quad \leq D n^{-\beta / \alpha} \int_{0}^{\infty}\left|f^{\prime}(x)\right| x^{-(\alpha+\beta)} e^{-c x^{-\alpha}} d x=\mathcal{O}\left(n^{-\beta / \alpha}\right) .
\end{aligned}
$$

- By taking $\epsilon=\epsilon_{n} \rightarrow 0$, and using a mild technical condition we can also bound the integral near zero

$$
\int_{0}^{\epsilon_{n}}\left|F_{n}(x)-G(x)\right|\left|f^{\prime}(x)\right| d x
$$

Proposition If $\sigma^{\alpha}(x) \sim D x^{-\beta}$, as $x \rightarrow \infty$, then as $n \rightarrow \infty$,

$$
n^{\beta / \alpha}\left(\mathbb{E} f\left(M_{n}\right)-\mathbb{E} f(Z)\right) \longrightarrow D \int_{0}^{\infty} x^{-(\alpha+\beta)} f^{\prime}(x) e^{-C x^{-\alpha}} d x
$$

provided mild technical conditions on $\sigma(x)$ at 0 and on $f$ at 0 and $\infty$ hold. 


\section{Rates (cont'd)}

- We have thus obtained exact rates for moment functionals

$$
\mathbb{E} f\left(\frac{1}{n^{1 / \alpha}} \max _{1 \leq i \leq n} X_{i}\right)
$$

- They are valid for a large class of absolutely continuous $f$, including:

$$
f(x)=\log _{2}(x), \quad \text { and } \quad f(x)=x^{p}, p \in(0, \alpha),
$$

for example.

- More details can be found in Stoev, Michailidis and Taqqu (2006).

- As a corollary, we also get rates of convergence for $\operatorname{Cov}\left(\log _{2} D\left(r+j_{1}, k\right), \log _{2} D\left(r+j_{2}, k\right)\right), \quad$ as $r=r(n) \rightarrow \infty$.

- These tools and the Berry-Esseen Theorem, yield the uniform asymptotic normality of the max-spectrum.

Thank you! 


\section{References}

Csörgő, S., Deheuvels, P. \& Mason, D. (1985), 'Kernel estimates of the tail index of a distribution', Annals of Statistics 13(3), 1050-1077.

Dekkers, A., Einmahl, J. \& de Haan, L. (1989), 'A moment estimator for the index of an extreme-value distribution', Ann. Statist. 17(4), 1833-1855.

Hall, P. (1982), 'On some simple estimates of an exponent of regular variation', J. Roy. Stat. Assoc. 44, 37-42. Series B.

Hill, B. M. (1975), 'A simple general approach to inference about the tail of a distribution', The Annals of Statistics 3, 1163-1174.

Pickands, J. (1975), 'Statistical inference using extreme order statistics', Ann. Statist. 3, 119-131.

Stoev, S., Michailidis, G. \& Taqqu, M. (2006), Estimating heavy-tail exponents through max self-similarity, Preprint.

Weissman, I. (1978), 'Estimation of parameters and large quantiles based on the $k$ largest observations', Journal of the American Statistical Association 73, 812-815. 